\title{
Measurement of the Time-like Pion Transition Form Factor at BESIII
}

\author{
Thomas Lenz ${ }^{1, *}$, Achim Denig ${ }^{1}$, and Christoph Florian Redmer ${ }^{1}$ \\ ${ }^{1}$ Institute of Nuclear Physics, Johannes Gutenberg University Mainz
}

\begin{abstract}
The pion transition form factor is an important input to the dispersive approaches of the Standard Model calculations for the anomalous magnetic moment of the muon. We report the prospects of a first measurement at high momentum transfer in the time-like region performed at BESIII. The aim is to improve the uncertainty of the hadronic light-by-light calculations and to shed light on the BaBar-Belle puzzle in the space-like region.
\end{abstract}

\section{The Anomalous Magnetic Moment of the Muon}

The anomalous magnetic moment of the muon $a_{\mu}=(g-2)_{\mu} / 2$ is one of the most precisely known observables in physics, both experimentally and theoretically. The result of the direct measurement at BNL [1] deviates by $3.6 \sigma$ from the Standard Model predictions [2]. This discrepancy might be a hint for contributions from New Physics.

With an expected improvement of the uncertainty of the direct measurement by a factor of four from Fermilab [3] and J-PARC [4], it is necessary to improve the uncertainty of the Standard Model calculations. The dominating part of these uncertainties arise from hadronic contributions: the hadronic light-by-light scattering (HLbL) and the hadronic vacuum polarization (HVP). Due to the running of the strong coupling constant $\alpha_{\mathrm{s}}$ both contributions cannot be determined pertubatively in the relevant energy regime.

Data-driven approaches use inputs from $e^{+} e^{-}$scattering experiments to improve Standard Model calculations based on dispersion theory. In case of HLbL, the coupling of two photons and a pseudoscalar meson is the most relevant subprocess, which is described by transition form factors (TFF). These can be measured and used to improve the theoretical uncertainty [5, 6]. Similarly, for the HVP calculations hadronic cross sections can be used as input [2,7].

\section{The Pion Transition Form Factor}

The TFF $\mathcal{F}_{\pi^{0}}\left(q_{1}^{2}, q_{2}^{2}\right)$ models the interaction of pseudoscalar mesons, like the $\pi^{0}$, with two photons. It depends on the momentum transfers $q_{1}^{2}$ and $q_{2}^{2}$ of the photons, which can be either time-like $\left(q^{2}>0\right)$ or space-like $\left(Q^{2}=-q^{2}>0\right)$.

In the space-like region the measurement is performed in $e^{+} e^{-}$collisions where the leptons interact by exchanging two photons, which in turn annihilate and produce pseudoscalar mesons. Several experiments have measured the TFF of $\pi^{0}$ [8-11], where one of the photons is quasi-real $\left(q^{2} \approx 0\right)$. The latest preliminary result from BESIII [12] is shown in Fig. 2.

\footnotetext{
*e-mail: lenz@uni-mainz.de
} 
In the time-like region the TFF has been measured using the reaction $e^{+} e^{-} \rightarrow \pi^{0} \gamma$ at SND [13] and CMD-2 [14], and using the Dalitz decay $\pi^{0} \rightarrow e^{+} e^{-} \gamma$ at A2 [15] and NA62 [16].

Pertubative QCD (pQCD) predicts an asymptotic behavior of the TFF for very high spacelike momentum transfer $Q^{2} \rightarrow \infty$, the Brodsky-Lepage limit [17]. The behavior is predicted for both, time- and space-like momentum transfer. Since the measurement suggested here will be the first time-like measurement at high momentum transfer, it allows to test this pQCD prediction. Furthermore, this measurement will also shed light on the BaBar-Belle puzzle in the space-like region where those two measurements do not fully agree on each other.

\section{The BESIII Experiment}

The BESIII experiment, described in detail in Ref. [18], is located and operated at the BEPCII accelerator facility at the Institute of High Energy Physics (IHEP) in Beijing, China. Centerof-mass energies $\sqrt{s}$ between $2.0 \mathrm{GeV}$ and $4.6 \mathrm{GeV}$ are provided and the design luminosity of $1.0 \times 10^{33} \mathrm{~cm}^{-2} \mathrm{~s}^{-1}$ at $\sqrt{s}=3.773 \mathrm{GeV}$ was achieved in April 2016 .

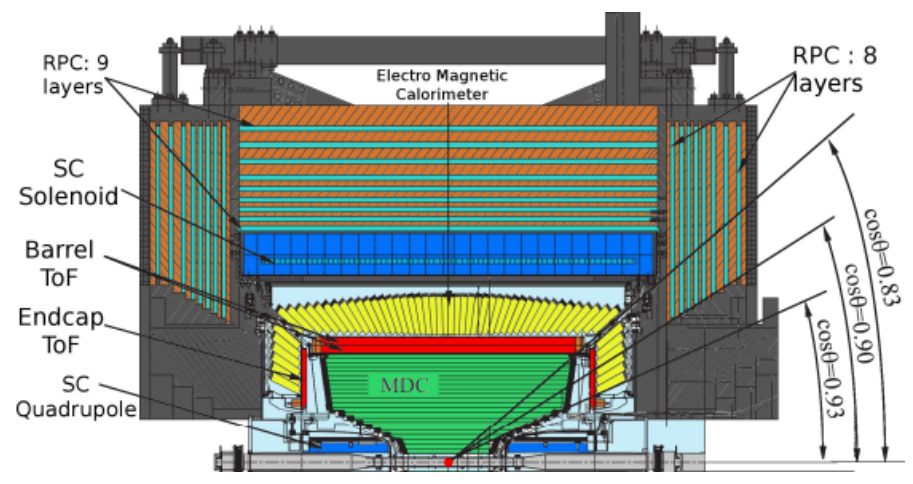

Figure 1. Schematic drawing of the top half of the BESIII detector [18].

The detector is shown in Fig. 1 and contains a multi-layer drift chamber (MDC), a timeof-flight (ToF) system, an electromagnetic calorimeter (EMC), a solenoid magnet with a $1 \mathrm{~T}$ magnetic field and resistive plate chambers (RPC) in the flux return yoke for muon identification.

\section{Analysis}

The time-like $\pi^{0}$ transition form factor $\mathcal{F}_{\pi^{0}}\left(q^{2}, 0\right)$ is measured in the reaction $e^{+} e^{-} \rightarrow \pi^{0} \gamma$. The momentum transfer $q^{2}=s$ of the virtual photon is fixed to the center-of-mass energy $\sqrt{s}$. The $\pi^{0}$ decays predominantly to two photons [19], hence, three photons will be detected in the detector in total.

\subsection{Data Sets and Simulation}

BESIII acquired large data sets with high statistics [20-23], which allows to perform the analysis on a combined data set of $9 \mathrm{fb}^{-1}$. The momentum transfer in this sample varies between $14.2 \mathrm{GeV}^{2}$ and $19.0 \mathrm{GeV}^{2}$, and the luminosity weighted average momentum transfer is $\bar{q}^{2}=(16.5 \pm 0.1) \mathrm{GeV}^{2}$. 
The signal channel $e^{+} e^{-} \rightarrow \pi^{0} \gamma$ is simulated using Phokhara 9.3 [24]. For the main background from $e^{+} e^{-} \rightarrow \gamma \gamma$ BABAYAGA $@$ NLO [25] is used. An inclusive Monte Carlo simulation is used to develop selection criteria and study further background contributions.

\subsection{Event Selection}

Since the signal channel only involves neutral particles, events with charged tracks are rejected. Furthermore, at least three good photon candidates are required. A cluster in the barrel part $(|\cos \theta| \leq 0.8)$ of the EMC with $E_{\gamma} \geq 0.025 \mathrm{GeV}$ is considered as good photon candidate. The pion is reconstructed by combining two photon candidates, which have an invariant mass between $0.1 \mathrm{GeV}$ and $0.2 \mathrm{GeV}$. All combinations are subjected to a kinematic fit, which is constrained by energy- and momentum-conservation. The combination with the lowest $\chi^{2}$ is selected.

Significant background rejection is achieved by a requirement on the helicity angle of the pion decay photons. The main sources of background are from $e^{+} e^{-} \rightarrow \gamma \gamma$ and from machine background.

\subsection{Photon Conversion Suppression}

Simulations suggest that the majority of the background events has only two neutral signals in the final state at generator level, while three are required in the event selection. A potential source of this background is photon conversion in the MDC. A photon creates an electronpositron pair, but the lepton tracks are not reconstructed due to inefficiencies of the pattern recognition algorithm. However, these events can still be identified, since the leptons induce signals in the MDC, which are spatially correlated to the detected clusters in the EMC. Counting hits in a volume of the MDC related to an energy deposition in the EMC can be used to identify the signal events.

\section{Summary and Outlook}

The measurement of the time-like $\pi^{0} \mathrm{TFF}$ at high momentum transfer at BESIII is feasible. It will be the first measurement at high momentum transfer and will be complementary to existing time- and space-like measurements at BESIII and other experiments. This measurement tests the predictions of pQCD and sheds light on the BaBar-Belle puzzle.

The analysis is on-going. A first analysis scheme is developed and the identified means of background suppression offer a potential application of machine learning for an improved photon conversion suppression and background reduction in general.

\section{References}

[1] G.W. Bennett et al. (Muon g-2), Phys. Rev. D73, 072003 (2006)

[2] A. Keshavarzi, D. Nomura, T. Teubner, Phys. Rev. D97, 114025 (2018)

[3] J. Grange et al. (Muon g-2) (2015), 1501.06858

[4] H. Iinuma (J-PARC Muon g-2/EDM), J. Phys. Conf. Ser. 295, 012032 (2011)

[5] G. Colangelo, M. Hoferichter, M. Procura, P. Stoffer, JHEP 09, 074 (2015)

[6] V. Pauk, M. Vanderhaeghen, Phys. Rev. D90, 113012 (2014)

[7] M. Davier, A. Hoecker, B. Malaescu, Z. Zhang, Eur. Phys. J. C77, 827 (2017)

[8] H.J. Behrend et al. (CELLO), Z. Phys. C49, 401 (1991) 


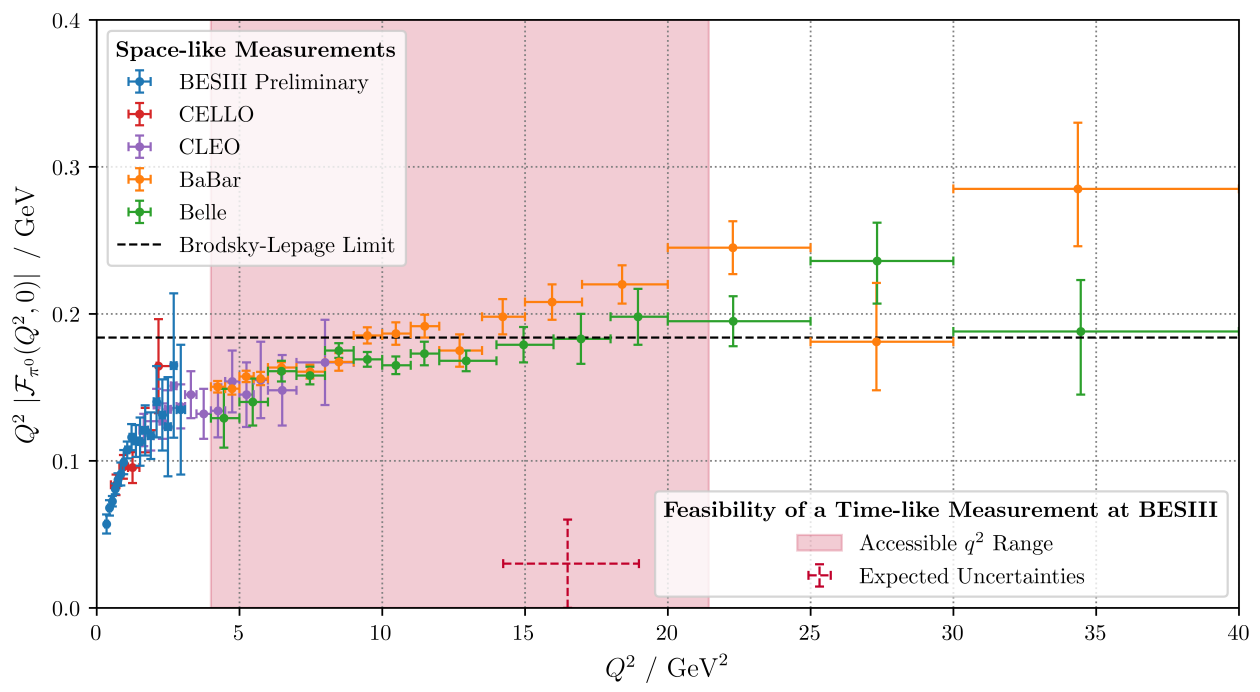

Figure 2. Measurements of the space-like $\pi^{0}$ TFF from BESIII (preliminary) [12], CELLO [8], CLEO [9], BaBar [10] and Belle [11] and the Brodsky-Lepage limit [17]. The red shaded area indicates the region of the time-like momentum transfer accessible at BESIII. The dashed error bars indicate the $q^{2}$ range and the expected statistically uncertainty of the combined data sets of this time-like measurement. The central value is chosen arbitrarily does not reflect the expected result.

[9] J. Gronberg et al. (CLEO), Phys. Rev. D57, 33 (1998)

[10] B. Aubert et al. (BaBar), Phys. Rev. D80, 052002 (2009)

[11] S. Uehara et al. (Belle), Phys. Rev. D86, 092007 (2012)

[12] C.F. Redmer (BESIII), Measurement of meson transition form factors at BESIII, in 13th Conference on the Intersections of Particle and Nuclear Physics (CIPANP 2018) Palm Springs, California, USA, May 29-June 3, 2018 (2018), 1810.00654

[13] M.N. Achasov et al. (SND), Phys. Rev. D93, 092001 (2016)

[14] R.R. Akhmetshin et al. (CMD-2), Phys. Lett. B605, 26 (2005)

[15] P. Adlarson et al. (A2), Phys. Rev. C95, 025202 (2017)

[16] C. Lazzeroni et al. (NA62), Phys. Lett. B768, 38 (2017)

[17] G.P. Lepage, S.J. Brodsky, Phys. Rev. D22, 2157 (1980)

[18] M. Ablikim et al. (BESIII), Nucl. Instrum. Meth. A614, 345 (2010)

[19] M. Tanabashi et al. (Particle Data Group), Phys. Rev. D98, 030001 (2018)

[20] M. Ablikim et al. (BESIII), Chin. Phys. C41, 013001 (2017)

[21] M. Ablikim et al. (BESIII), Chin. Phys. C42, 023001 (2018)

[22] M. Ablikim et al. (BESIII), Chin. Phys. C37, 123001 (2013)

[23] M. Ablikim et al. (BESIII), Chin. Phys. C39, 093001 (2015)

[24] H. Czyz, P. Kisza, S. Tracz, Phys. Rev. D97, 016006 (2018)

[25] C. Carloni Calame, H. Czyz, J. Gluza, M. Gunia, G. Montagna, O. Nicrosini, F. Piccinini, T. Riemann, M. Worek, JHEP 07, 126 (2011) 